\title{
Hemorragia digestiva en paciente con hiperplasia de glándulas de Brunner
}

\section{Digestive tract hemorrhaging in a patient with Brunner's gland hyperplasia}

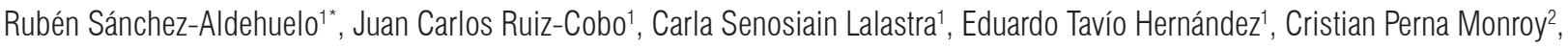 \\ Agustín Albillos ${ }^{1}$
}

\footnotetext{
1 Servicio de Gastroenterología y Hepatología, Hospital Universitario Ramón y Cajal, Madrid, España.

2 Servicio de Anatomía Patológica, Hospital Universitario Ramón y Cajal, Madrid, España.

*Correspondencia: Rubén Sánchez-Aldehuelo, ruben.sanchez.aldehuelo@gmail.com

Fecha recibido: $\quad 24 / 05 / 18$ Fecha aceptado: $01 / 07 / 18$
}

\begin{abstract}
Resumen
Objetivo: analizar las características epidemiológicas, bases etiopatogénicas y presentación clínica, así como el diagnóstico y el tratamiento de la hiperplasia de glándulas de Brunner (HGB). Métodos: describir un caso de HGB diagnosticado de forma incidental durante una endoscopia electiva y realizar una revisión de la literatura disponible hasta el momento. Resultados: esta neoformación consiste en una proliferación glandular localizada preferentemente en el duodeno proximal. Su diagnóstico, normalmente realizado mediante biopsia endoscópica, puede asociarse con complicaciones que, aunque infrecuentes, no deben ser subestimadas. Conclusiones: las neoplasias duodenales representan un porcentaje pequeño dentro del total de las que afectan al tracto gastrointestinal. Debido a que el diagnóstico de estas lesiones suele realizarse de forma casual durante una endoscopia programada, el tratamiento deberá basarse en la sintomatología, así como el tamaño de las mismas, de acuerdo con los estándares de tratamiento de cada centro.
\end{abstract}

\section{Palabras clave}

Hemorragia digestiva, glándulas de Brunner, hiperplasia de glándulas de Brunner, adenoma de glándulas de Brunner.

\begin{abstract}
Objective: This study analyzes the epidemiological characteristics, etiological and pathogenic bases, clinical presentation, diagnosis and treatment of Brunner's gland hyperplasia. Methods: We describe a case of Brunner's gland hyperplasia that was diagnosed incidentally during elective endoscopy and review the available literature. Results: This neoplasm consists of glandular proliferation preferentially located in the proximal duodenum. Its diagnosis, normally made by endoscopic biopsy, can be associated with complications that, although infrequent, should not be underestimated. Conclusions: Duodenal neoplasms are a small percentage of those that affect the gastrointestinal tract. Because diagnosis is usually made by chance during a scheduled endoscopy, treatment should be based on the symptoms and size of the lesion according to the treatment standards of each medical center.

\section{Keywords}

Digestive hemorrhage, Brunner's glands, Brunner's gland hyperplasia, Brunner's gland adenoma.
\end{abstract}

\section{INTRODUCCIÓN}

La hiperplasia de glándulas de Brunner (HGB), también conocida como adenoma de glándulas de Brunner o Brunneroma, consiste en una proliferación de estas glán- dulas submucosas de localización preferentemente duodenal, de tamaño y morfología variable. Su prevalencia no está bien establecida, ya que en la mayoría de los casos son lesiones descubiertas de forma incidental durante una exploración endoscópica. Aunque normalmente son asin- 
tomáticas, estas pueden producir una variada sintomatología gastrointestinal, que abarca desde náuseas y vómitos hasta hemorragia digestiva, frecuente por su profusa vascularización. El diagnóstico se lleva a cabo mediante endoscopia, ecoendoscopia u otras técnicas de imagen; mientras que el tratamiento, en caso de resultar necesario, debe realizarse de forma individualizada, ya sea por vía endoscópica o quirúrgica.

\section{EXPOSICIÓN DEL CASO CLÍNICO}

Paciente masculino de 60 años, fumador y exconsumidor de 30 gramos de alcohol diarios, con antecedentes de hipertensión arterial, enfermedad renal crónica estadio IV, secundaria a nefroangioesclerosis, y hepatopatía crónica de origen enólico, sin datos de hipertensión portal. Recibe como tratamiento habitual espironolactona $25 \mathrm{mg} /$ día y enalapril $40 \mathrm{mg} /$ día.

Acude a la unidad de endoscopias para realizar una endoscopia digestiva alta (EDA) para cribado de varices. Durante la exploración se identifica, en la segunda porción duodenal distalmente a la papila duodenal, dos lesiones nodulares de aspecto subepitelial de unos $10 \mathrm{~mm}$ recubiertas de mucosa normal (Figura 1). Con intención diagnóstica se realiza biopsia sobre biopsia de una de ellas, lo que provoca un sangrado babeante persistente, que requiere colocar un hemoclip para conseguir hemostasia. No se identifican otras lesiones ni datos de hipertensión portal.

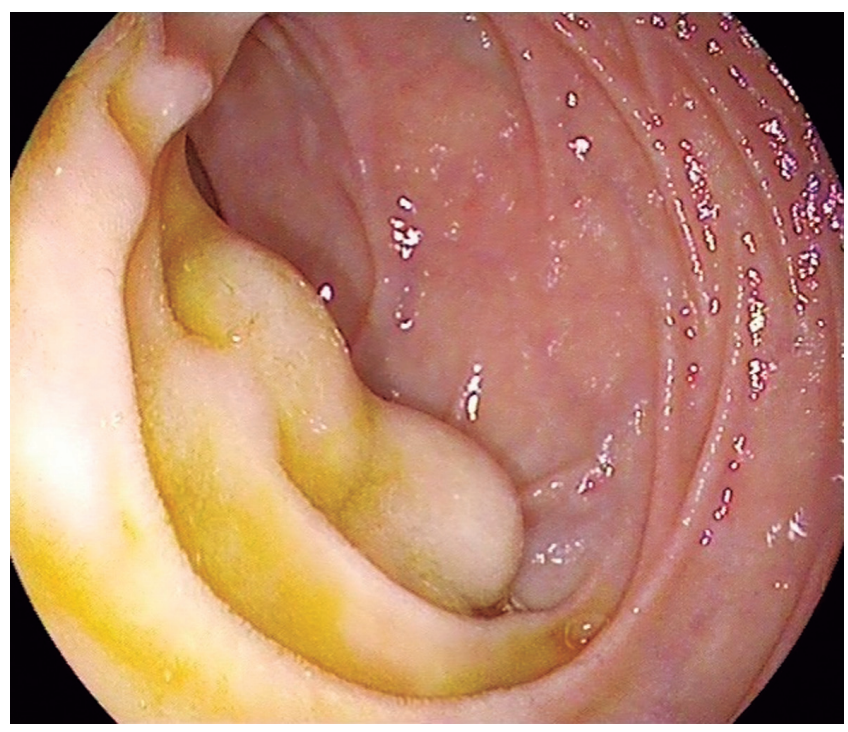

Figura 1. Dos lesiones de aspecto subepitelial recubiertas de mucosa normal inmediatamente distales a la papila duodenal.

Luego de 4 días, el paciente acude al servicio de Urgencias presentando deposiciones melénicas de 2 días de evolución. A su llegada se encuentra estable hemodinámicamente, con valores de tensión arterial de 110/80 mm $\mathrm{Hg}$ y frecuencia cardíaca de 73 latidos por minuto. En la analítica destaca anemización significativa, con un valor de hemoglobina de 9,7 $\mathrm{g} / \mathrm{dL}$ (previa de $14,3 \mathrm{~g} / \mathrm{dL}$ ), con el resto de los parámetros en sus valores habituales. Ante la sospecha de hemorragia digestiva alta se inicia tratamiento intravenoso con inhibidores de la bomba de protones en bolo y perfusión continua y se realiza EDA urgente. En segunda porción duodenal, en el lugar donde se llevaron a cabo las biopsias, se identifica un coágulo adherido al hemoclip previamente colocado, sin sangrado activo. Se coloca nuevo hemoclip, lo que produjo de nuevo hemorragia abundante, por lo que se realiza esclerosis con $10 \mathrm{~mL}$ de adrenalina diluida en suero salino fisiológico al $0,9 \%(1: 10000)$ y $2 \mathrm{~mL}$ de Etoxiesclerol ${ }^{\oplus}$ (lauromacrogol $400)$ y se colocan 2 hemoclips, lo que consigue detener el sangrado. En el hemograma de control se evidencia descenso de hemoglobina hasta $7,5 \mathrm{~g} / \mathrm{dL}$, por lo que se transfunden 2 concentrados de hematíes sin complicaciones ulteriores y se procede al alta a los 5 días.

En el estudio histológico de la lesión se observa proliferación de glándulas submucosas, por lo que se diagnostica hiperplasia de glándulas de Brunner (Figura 2). La tomografía computarizada (TC) mostró ausencia de extensión extraluminal. Dado el pequeño tamaño de la misma, el origen iatrogénico de la hemorragia y la ulterior ausencia de sintomatología se decide actitud expectante y el paciente permanece asintomático 8 meses después del diagnóstico.

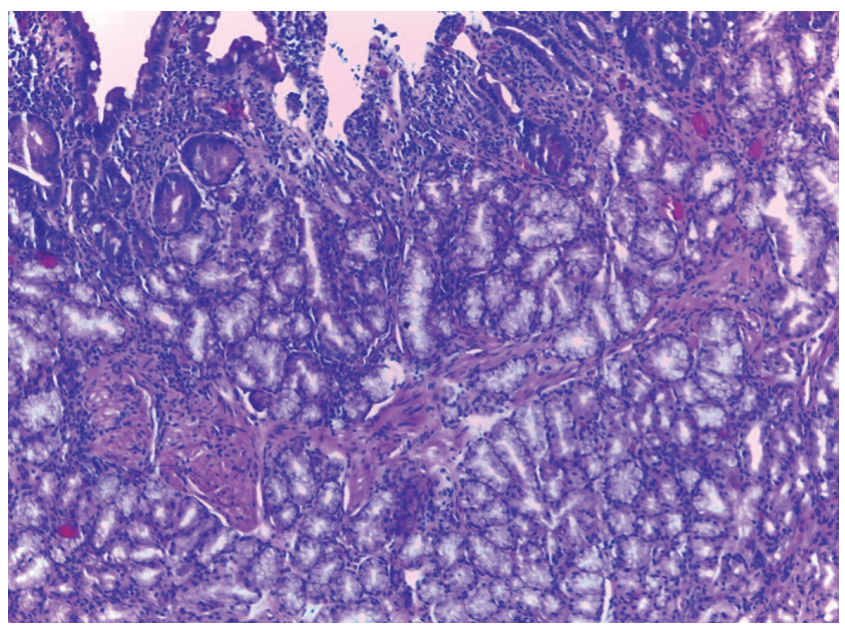

Figura 2. En la mucosa se observa una altura vellositaria correcta y lóbulos de glándulas de Brunner en más de la mitad de la misma. Estos lóbulos se continúan con abundantes glándulas de Brunner a nivel submucoso. Las células muestran el típico citoplasma claro mucoso, sin observarse atipia citológica (H\&E x400).

\section{DISCUSIÓN}

Las glándulas de Brunner son estructuras de morfología acinotubular y localización submucosa. Se sitúan principal- 
mente en el duodeno proximal, entre el píloro y la papila duodenal, si bien también pueden encontrarse distribuidas en menor medida en porciones más distales del duodeno e incluso en el yeyuno. Su función fundamental consiste en la producción y secreción de moco alcalino, que ayuda a proteger la mucosa duodenal del quimo procedente del estómago $(1,2)$. Aunque no existe un acuerdo establecido en cuanto a la nomenclatura de estas proliferaciones glandulares, denominadas en su conjunto hiperplasia de glándulas de Brunner, podemos encontrar tanto hiperplasia difusa $(<1 \mathrm{~cm})$ como lesiones polipoideas $(>1 \mathrm{~cm})$, comúnmente conocidas como Brunneromas (3). Dentro de estas últimas podemos diferenciar entre hamartoma y adenoma, según posean o no elementos mesenquimales (2).

Aunque la etiopatogenia de estas lesiones no está claramente establecida, existen varias teorías que intentan explicar su formación, siendo la hipótesis de la hiperclorhidria una de las más aceptadas. La sobreproducción de ácido gástrico actuaría como estímulo para la proliferación compensadora de las glándulas de Brunner, que finalmente culmina en una HGB (4). Otras hipótesis sitúan su origen en lesiones disembrioplásicas, mientras que otras relacionan su aparición como una manera de adaptación a procesos inflamatorios locales (infección por $H$. pylori o pancreatitis crónica) $(1,2)$.

Aparece predominantemente en pacientes de edad media, sin diferencias en cuanto a la distribución por sexos (5). De forma general, los tumores primarios del duodeno son poco frecuentes y representan menos del $1 \%$ de los tumores gastrointestinales. La HGB podría tener una incidencia del $0,008 \%$ según algunos estudios (5). No obstante, resulta difícil establecer su prevalencia, ya que la mayoría de las veces son lesiones asintomáticas encontradas incidentalmente en la endoscopia y solo una pequeña proporción de ellas se diagnostica por la clínica (6-8).

De forma general, los pólipos de mayor tamaño tienden a presentar síntomas con mayor frecuencia, siendo los más habituales algunos inespecíficos, como náuseas, vómitos o dolor abdominal crónico. Otros menos frecuentes son la hemorragia digestiva en forma de sangre oculta en heces o melenas (debido a su profusa vascularización), pancreatitis recidivante, ictericia obstructiva e incluso fístulas biliares $(6,9)$. Con respecto al potencial maligno, Sakurai y colaboradores observaron displasia en $2,1 \%$ y carcinoma invasivo en $0,3 \%$ de los 722 casos de HGB estudiados (10).

Para establecer el diagnóstico de esta entidad, la prueba de primera elección es la EDA con toma de biopsias; sin embargo, dada la naturaleza submucosa de estas glándulas, con frecuencia las muestras histológicas obtenidas mediante biopsia convencional son negativas o no concluyentes, siendo necesarios métodos de biopsia más profundos, como la biopsia sobre biopsia o la polipectomía, que ofrecen mayor rentabilidad, aunque también se asocian con mayor riesgo de complicaciones, como hemorragia o perforación (9).

En caso de histología no concluyente, la ecoendoscopia es la técnica de elección para la caracterización de estas lesiones subepiteliales, lo que permite determinar su origen y estudiar su vascularización $(1,3)$. Por último, algunos autores proponen la TC con contraste intravenoso para establecer tanto la extensión como el diagnóstico diferencial con otras entidades, como pólipo adenomatoso, lipoma, leiomioma, tumor del estroma gastrointestinal (GIST), entre otros. De esta forma se consigue evitar tratamientos excesivamente agresivos en estas lesiones de bajo potencial maligno, así como planificar un adecuado abordaje terapéutico $(2,3)$.

En cuanto al tratamiento, no existen guías clínicas ni estudios de calidad al respecto. Se acepta que estas lesiones deben ser resecadas cuando tienen un tamaño mayor de 2 $\mathrm{cm}$, así como cuando producen síntomas (2). El abordaje terapéutico puede realizarse por vía endoscópica o quirúrgica y se debe individualizar cada caso en función de las características del paciente, la lesión y la experiencia del centro. Hasta el momento no existe evidencia de recurrencia tras su extirpación mediante cualquiera de los dos métodos. En los últimos años se ha experimentado un auge en la tendencia al tratamiento por vía endoscópica debido a la menor morbimortalidad asociada, propiciada por los avances técnicos. Entre las técnicas endoscópicas se encuentran la resección con asa de polipectomía tradicional, mediante endoloop o disección endoscópica submucosa (2). Desde el punto de vista quirúrgico, las técnicas abarcan desde la polipectomía transduodenal hasta la duodenopancreatectomía.

\section{CONCLUSIÓN}

El diagnóstico de las lesiones duodenales, como la HGB, normalmente se alcanza tras el estudio histológico de las biopsias obtenidas durante la endoscopia, siendo este un procedimiento no exento de potenciales complicaciones, como la hemorragia digestiva, debido a su abundante irrigación vascular. El tratamiento de esta entidad, si fuera necesario, se realiza de forma individualizada, ya sea por vía endoscópica o quirúrgica.

\section{Conflicto de intereses}

Los autores declaran no tener ningún conflicto de intereses.

\section{REFERENCIAS}

1. Lu L, Li R, Zhang G, Zhao Z, Fu W, Li W. Brunner's gland adenoma of duodenum: report of two cases. Int J Clin Exp Pathol. 2015;8(6):7565-9. 
2. Sorleto M, Timmer-Stranghöner A, Wuttig H, Engelhard O. Brunner's gland adenoma - A rare cause of gastrointestinal bleeding: case report and systematic review. Case Rep Gastroenterol. 2017;11(1):1-8. doi: https://doi. org/10.1159/000454711.

3. Peloso A, Viganò J, Vanoli A, Dominioni T, Zonta S, Bugada D, et al. Saving from unnecessary pancreaticoduodenectomy. Brunner's gland hamartoma: case report on a rare duodenal lesion and exhaustive literature review. Ann Med Surg. 2017;17:43-9. doi: https://doi.org/10.1016/j. amsu.2017.03.034.

4. Franzin G, Musola R, Ghidini O, Manfrini C, Fratton A. Nodular hyperplasia of Brunner's glands. Gastrointest Endosc. 1985;31(6):374-8. doi: https://doi.org/10.1016/ S0016-5107(85)72251-1.

5. Levine JA, Burgart LJ, Batts KP, Wang KK. Brunner's gland hamartomas: clinical presentation and pathological features of 27 cases. Am J Gastroenterol. 1995;90(2):290-4.

6. Van de Walle P, Dillemans B, Vandelanotte M, Proot L. The laparoscopic resection of a benign stromal tumour of the duodenum. Acta Chir Belg. 1997;97(3):127-9.
7. Ohba R, Otaka M, Jin M, Odashima M, Matsuhashi T, Horikawa $\mathrm{Y}$, et al. Large Brunner's gland hyperplasia treated with modified endoscopic submucosal dissection. Dig Dis Sci. 2006;52(1):170-2. doi: https://doi.org/10.1007/ s10620-006-9607-1.

8. Botsford TW, Crowe P, Crocker DW. Tumours of the small intestine. A review of experience with 115 cases including a report of a rare case of malignant hemangio-endothelioma. Am J Surg. 1962;103:358-65. doi: https://doi. org/10.1016/0002-9610(62)90226-X.

9. Jung Y, Chung IK, Lee TH, Cho YS, Jo YG, Park SH, et al. Successful endoscopic resection of large pedunculated Brunner's gland hamartoma causing gastrointestinal bleeding arising from the pylorus. Case Rep Gastroenterol. 2013;7(2):304-7. doi: https://doi. org/10.1159/000354138.

10. Sakurai T, Sakashita H, Honjo G, Kasyu I, Manabe T. Gastric foveolar metaplasia with dysplastic changes in Brunner gland hyperplasia: possible precursor lesions for Brunner gland adenocarcinoma. Am J Surg Pathol. 2005;29:1442-8. doi: https://doi.org/10.1097/01.pas.0000180449.15827.88. 